\title{
Chronic Oxidative Stress and Comorbidities in the HIV-1 Transgenic Rat
}

\author{
Frank Denaro $^{1^{*}}$, Myla Worthington ${ }^{1}$, Francesca Benedetti ${ }^{2}$, Sabrina Curreli ${ }^{2}$, Davide Zella ${ }^{2}$, Joseph \\ Bryant $^{2}$ \\ 1. Morgan State University, Department of Biology, Baltimore, MD, USA. \\ 2. Institute of Human Virology, University of Maryland Medical Center, Baltimore, MD, USA. \\ * Corresponding author: frank.denaro@morgan.edu
}

The pathogenesis of AIDS comorbidities are the result of a number of mechanisms that can ultimately lead to cell death. One such mechanism for which there is accumulating evidence is chronic oxidative stress (COS). The impact of COS is not limited only to HIV infected cells. Uninfected cells have been shown to experience COS in patients and models of AIDS. Affected cells can have altered physiology and can die early. This makes the impact of COS far reaching, because the HIV reservoir cells are not the only cell types which are dying or displaying altered functions. This mechanism is believed to be dependent on the HIV proteins (for example: GP-120, Tat) that the infected cells release. These viral products have been demonstrated to cause oxidative stress in experimental cell cultures. Importantly, the process of COS has given insight to the comorbidities that are becoming more evident as the patients age. Currently, antiviral treatment is very effective and has improved and extended life. But even with the elimination of most opportunistic infections and keeping the HIV viral load very low, various tissues still display evidence of pathology. It is believed that the low levels of the viral proteins produced by the reservoirs, (which are not eliminated by the antivirals), produce the pathology slowly over time. This is why the magnitude of the comorbidities develop as the patient ages. It also explains why some non-infected cell types display pathology.

There are a number of organs/tissues that display pathology and altered function. It was recognized early in the AIDS epidemic that the brain, kidney, heart and endothelium displayed acute pathological changes as well as long term ones. Analysis revealed that HIV infected cells were of low frequency in these tissues. When studies have ruled out opportunistic infections, other mechanisms were sought to explain the pathology. One mechanism is inflammation with a "bystander effect". But, Anti-viral therapy resulted in a noted drop of infected cells, and inflammatory cell infiltrates also became more infrequent. Therefore, the toxic characteristics of the HIV viral proteins are now a focus for the generation of HIV comorbidities.

We have developed a noninfectious transgenic model which produces HIV-1 viral proteins [1]. In this model GP-120, Tat and Nef have been identified in cells of the immune system. Also, Gp-120 has been identified and measured in sera and CSF. The release of viral proteins into the blood provide a means by which many cell types can be exposed to those viral proteins. To identify COS, we initially used an immunocytochemical marker for Nitrotyrosine (NT) which can detect the interaction of nitric oxide with tyrosine [2]. With this marker, we were able to identify NT in the brains of HIV infected Humans, monkeys and the HIV-1 transgenic rat [2]. Further analysis of the HIV-1 transgenic rat revealed their pathology is a time-dependent process. This is similar to the evolution of the pathology in humans [3]. In the present study we sought to further characterize the cell types and locations of NT labeling cells in the brain, heart, kidney and endothelia cells. The present survey in the HIV Tg Rat reveals that NT labeling can be found in tissues and organs which displays mark pathology, consistent with HIV infection. Moreover, these tissues display evidence for early senescence [3]. Evidence for the role of COS in long term HIV infected patients is becoming stronger. This suggests that the HIV-1 TG rat may play important 
part in clarifying the mechanisms of COS. Immunocytochemistry for Nitrotyrosine in tissues of the HIV1 Transgenic Rat are displayed in the following ten figures [4].

\section{References:}

[1] W Reid et al., Proceedings of the National Academy of Science 98 (2001), p.9271.

[2] FJ Denaro et al., Institute of Human Virology Annual Meeting (2000), p. 254.

[3] S Davinelli et al., Biogerontology 15 (2014), p. 449.

[4] The authors acknowledge funding from Grant numbers 5UL1GM118973, 1 R29 NS31857-01A1.
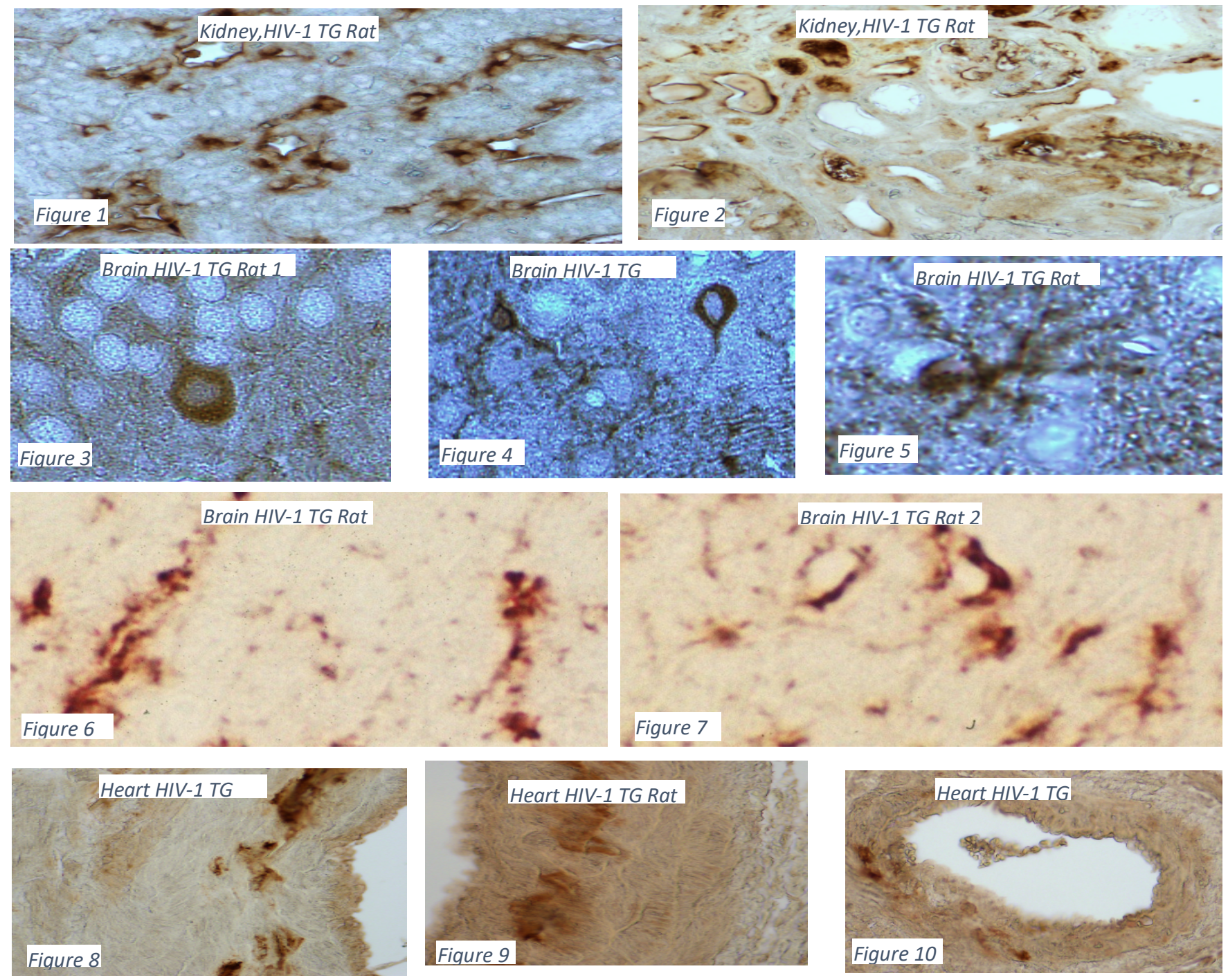

Figures 1-10. Immunostaining of NT. Reaction product brown in Figs 1,2,3,4,5,8,9 and red in Fig.6,7. Fig. 1 and 2 are Kidney. Dark brown staining can be found in the area of the macula densa. The renal glomeruli and the convoluted tubules. Figs 3-5 are brain. Fig. 3 displays a neuron in the hippocampus. Fig. 4 a neuron in the striatum. Fig. 5 a positive glia cell. Fig. 6 Red label of endothelia cells of the brain in longitudinal section. Fig 7, Red label of endothelia cells in transverse section. Figures 8-10 are the heart. The brown label can be found in the heart muscle and vessels. In the larger arteries the vasa vasorum appear labeled. 